Itinéraires Itinéraires

Littérature, textes, cultures

2014-3 | 2015

Le polar en Europe : réécritures du genre

\title{
De la « Noire » à la « Blanche » : la position mouvante du roman policier au sein de l'institution littéraire
}

\section{Marc Lits}

\section{CpenEdition}

Journals

Édition électronique

URL : https://journals.openedition.org/itineraires/2589

DOI : $10.4000 /$ itineraires.2589

ISSN : 2427-920X

Éditeur

Pléiade

\section{Référence électronique}

Marc Lits, « De la « Noire » à la « Blanche » : la position mouvante du roman policier au sein de l'institution littéraire », Itinéraires [En ligne], 2014-3 | 2015, mis en ligne le 25 septembre 2015, consulté le 07 décembre 2022. URL : http://journals.openedition.org/itineraires/2589 ; DOI : https://doi.org/ 10.4000/itineraires.2589

Ce document a été généré automatiquement le 29 septembre 2020.

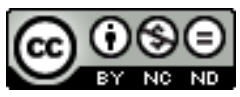

Creative Commons - Attribution - Pas d'Utilisation Commerciale - Pas de Modification 4.0 International - CC BY-NC-ND 4.0

https://creativecommons.org/licenses/by-nc-nd/4.0/ 


\title{
De la « Noire » à la «Blanche » : la position mouvante du roman policier au sein de l'institution littéraire
}

\author{
Marc Lits
}

1 Le journal Le Monde daté du 5 novembre 2013 annonce en ces termes l'attribution du nouveau prix Goncourt: «le roman de Pierre Lemaitre [...] était depuis plusieurs semaines le favori des pronostics pour le prix Goncourt. Il marque le passage à la littérature "blanche" de cet auteur de polars reconnu, âgé de 62 ans, traduit en vingt langues ». Le quotidien La Libre Belgique du même jour titre ainsi la biographie de l'auteur consacré : «Venu du roman noir ». L'hebdomadaire Paris Match, dans son interview du romancier primé publié deux jours plus tard, le 7 novembre, revient sur ce passage de « la noire » à « la blanche » :

- Pourquoi vouloir quitter l'univers des séries noires, justement?

- Quand on est un auteur noir, qu'on écrit des polars, il arrive un moment, dans notre carrière, où l'on a envie de "sniffer de la blanche ": d'écrire des romans et de devenir, enfin, un écrivain. Je ressentais ce besoin. Aussi celui d'être reconnu par mes pairs. Avoir un prix littéraire, c'était pour moi une légitimité, une reconnaissance. On en rêve tous. Moi le premier.

- On qualifie votre roman de "populaire », « lisible », " grand public ». Ces mots vous blessent-ils?

- Absolument pas! Au contraire, je suis moi-même un lecteur populaire. J'aime Hector Malot, Alexandre Dumas... Je suis d'abord un auteur de polars, alors être labellisé « écrivain populaire » par le Goncourt est une grande fierté ${ }^{.}$.

Si l'on peut discuter du poids actuel des prix littéraires comme acteurs de la reconnaissance institutionnelle d'auteurs ou de genres, et de leur dimension de sélection dans la sphère restreinte des auteurs consacrés, qui n'est plus aussi forte que le disait Jacques Dubois (1988a) il y a près de trente ans (entre autres parce que le poids du marketing devient aussi déterminant que celui des critères esthétiques portés par les membres du jury), on ne peut négliger le signal donné par ce type de choix quant à des porosités grandissantes entre catégories littéraires autrefois bien distinctes. Ce dernier 
exemple s'ajoute à une longue liste d'écrivains qui étaient catalogués comme auteurs de romans policiers et qui se font reconnaître ensuite par des publications plus « légitimes ", soit parce qu'ils accèdent à des collections réputées, soit parce qu'ils reçoivent un prix littéraire comme Jean Vautrin avant Pierre Lemaître, quand il reçut le prix Goncourt en 1989 pour Un grand pas vers le bon Dieu. Ce qui pose à nouveau la question des limites des rapports ambigus entre le roman policier et la littérature consacrée et de l'interpénétration des catégories hiérarchiques au sein de l'institution littéraire.

3 Si l'objet « littérature » n'est plus aujourd'hui considéré comme un donné existant in se, il reste cependant un cadre très contraignant par rapport auquel les chercheurs qui s'intéressent à la paralittérature doivent sans cesse se situer. En effet, celui qui étudie ce type de production doit accepter de placer son objet d'étude, qu'on l'appelle « littérature populaire » ou "paralittérature », dans une logique de subordination par rapport à la littérature légitimée. Ce lien d'allégeance est sans doute moins marqué qu'il y a quelques décennies, mais il n'a pas encore disparu. Prenons par exemple cette critique de la collection « Le Poulpe » publiée dans un numéro de la revue Critique :

La série du Poulpe appartient au roman policier et peut être rangée dans ce que la critique appelle la paralittérature. Sous-littérature ou à côté de la littérature, la paralittérature accueille des romans juste bons à divertir : ils meubleront le temps d'un trajet ferroviaire entre Caen et Tours pour être ensuite abandonnés sur la banquette du compartiment, sans regrets. Pas plus que le roman sentimental ou d'espionnage, le polar n'a sa place dans une bibliothèque « sérieuse ». Son mode de diffusion, sa présentation, le discours qui l'accompagne sont autant de preuves qu'il n'a d'autre valeur que commerciale. (Lomnitz 1999 : 445)

L'article ne date pas d'il y a un siècle, mais de mai 1999. Cet exemple est emblématique des jugements de valeur qui imprègnent encore une certaine partie de la critique, inscrite dans une conception aristotélicienne de la littérature dissociant les genres nobles et vulgaires et reléguant les genres illégitimes dans les bas-fonds des champs de grande production ${ }^{2}$. Ces diktats obligent les chercheurs en littérature populaire à sans cesse justifier la légitimité sociale et culturelle de leur objet d'étude. Les critères définitoires de l'objet commun restent empreints de repères esthétiques, lesquels sont uniquement déterminés par rapport aux œuvres appartenant au champ de production restreinte. Il n'y a donc pas de reconnaissance ontologique des productions paralittéraires selon leurs critères propres (narrativité, sérialité, fonction de divertissement, modes de consommation, etc.), et même les tentatives de définir la paralittérature selon ses traits spécifiques, comme l'a réalisé d'excellente manière Daniel Couégnas (1992), échouent à autonomiser ce secteur. Et le fait même de vouloir l'autonomiser pose déjà problème. Mais il faut cependant faire la part entre les écrivains eux-mêmes et les critiques de presse, d'une part, qui semblent avoir dépassé ces clivages et toute forme d'ostracisme, et une critique universitaire, d'autre part, encore imprégnée majoritairement, pour le monde francophone, par les formes de distinction bourdieusienne, même quand cette critique se positionne sur des critères esthétiques plutôt que sociologiques.

\section{L'artisan contre le créateur}

5 Ce qui pose question pour les chercheurs a cependant été intégré par les auteurs de policiers, dans un premier temps. Thomas Narcejac avait posé très clairement la question fondamentale, dès 1975, et il y répondait sans ambages, tout auteur de romans policiers qu'il fut. Son essai s'ouvrait sur l'interrogation suivante : « Pourquoi dit-on souvent que le 
roman policier est un genre "paralittéraire" ?» (Narcejac 1975 : 15). Et le dernier chapitre du livre apportait la réponse : «Le feuilleton est une marchandise. Tant pis pour nous, auteurs policiers. Nous sommes ce que nous sommes. Ne confondons pas adresse et talent. Il faut toujours dire la vérité » (Ibid. : 247). On pourrait bien sûr lui rétorquer la citation de Bertolt Brecht (1970 : 78) :

Le fait qu'une caractéristique du roman policier consiste à exécuter des variations sur des éléments plus ou moins constants élève le genre tout entier au niveau esthétique. C'est un des signes auxquels on reconnait une branche cultivée de la littérature.

6 Les deux positions sont intéressantes à confronter, dans la mesure où Thomas Narcejac a intégré dans sa posture les critères déligitimants posés par les tenants du champ de production restreinte là où Bertolt Brecht revendique la spécificité d'une esthétique particulière. Bourdieu contre Couégnas, en quelque sorte.

On peut cependant constater que Narcejac fonde son exclusion du roman policier hors $\mathrm{du}$ champ littéraire en se rattachant à une conception de l'écrivain autant mythique qu'historiquement construite, telle que Claude Abastado a pu l'étudier pour des auteurs de la seconde moitié du XIX ${ }^{\mathrm{e}}$ siècle. Pour Narcejac, il y a une distinction nette à effectuer avec la littérature, « la vraie littérature, celle qui produit des "objets" parfaits [...], Racine, Shakespeare, Proust... Ici, le personnage détruit l'histoire, se nourrit à ses dépens, pour exister d'une vie qui lui est propre, qu'il a arrachée à son auteur ». Face à cette littérature authentique, il y a « la littérature de consommation, dont le roman policier est sans doute le modèle le plus achevé. [...] Ici, c'est le lecteur qui détruit l'histoire et se nourrit à ses dépens, simplement pour passer le temps" (Narcejac 1975: 246). Il y a en fait pour Narcejac une grande différence entre ces deux types de littérature, car si le véritable écrivain possède le génie créateur, le feuilletoniste ne dispose que d'habileté.

Si les auteurs de romans policiers ont tellement intégré cette distinction entre littérature de création et littérature de consommation massive, c'est aussi parce que l'institution elle-même s'est évertuée à maintenir cette dichotomie, comme l'a pleinement vécu Georges Simenon. Dans les Mémoires intimes, il déclare :

Je garde mes distances, d'instinct, avec les revues, magazines, journaux littéraires comme avec les associations ou groupements qui se réclament de ce mot. Je me sens, en effet, à l'opposé du "littéraire ", presque toujours féru de la forme et dédaigneux de la vie qui n'est pas passée par la moulinette des philosophes et des penseurs. (Simenon $1981: 456$ )

Cette distance, il ne l'a pas toujours respectée, puisqu'il a longtemps cherché à être accepté par ses « chers confrères » en littérature. Mais il comprit finalement que le succès public ne va pas toujours de pair avec la reconnaissance du milieu intellectuel. Il a d'emblée un projet très clairement annoncé: "Gagner le plus d'argent possible en écrivant des livres faciles, puis s'installer et faire de la littérature " (Assouline 1992: 99). Très vite, il accède à une certaine reconnaissance en tant qu'auteur de littérature populaire, avec toutes les caractéristiques de ce type de produit: une production très abondante, recourant à divers pseudonymes, diffusée dans des collections à bas prix, sous forme de fascicules, chez Ferenczi ou Tallandier. Ces volumes courts l'enferment dans un créneau qui ne lui assure pas de reconnaissance d'auteur; il va donc passer (si l'on accepte les ambiguïtés, limites et confusions des deux termes) de la littérature populaire, encore inscrite dans des modes de production et de consommation hérités du XIX siècle, à la paralittérature, en proposant désormais sous son nom des romans plus longs se réclamant d'un genre qui a plus de légitimité que ses romans polissons. Ce choix ne doit 
rien au hasard, puisque de tous les genres paralittéraires, le roman policier apparait comme un des plus acceptables par l'institution littéraire. C'est un espace de transition idéal pour accéder à la «vraie » littérature, d'autant que Simenon ne s'inféodera jamais aux règles canoniques du genre, même s'il y trouve un modèle narratif très fécond. Dans Mémoires intimes, il reconnaît que les Maigret ne sont qu'un passage :

Deux ans plus tard, quand la série de ces romans commencerait à paraître mensuellement, je ne serais plus un apprenti mais un romancier, un véritable professionnel. Et deux ans plus tard encore, je me libérais du roman policier pour écrire les romans qui naîtraient en moi. (Simenon $1981: 29$ )

Simenon a une vision très lucide du déroulement de sa carrière, et il comprend vite qu'il faut maîtriser les règles du jeu institutionnel pour accéder à ce que Pierre Bourdieu appelle le champ de production restreinte (Bourdieu 1991). Il a besoin de l'adoubement d'un maître pour être accepté par le milieu, et ce sera le rôle qu'il attribue à Gide auquel il s'adresse toujours avec la formule "Mon cher Maître ». Il s'agit aussi d'occuper un positionnement éditorial dans un lieu reconnu, ce qui sera atteint quand il passera de Fayard à Gallimard. Il faut encore obtenir une reconnaissance critique, qu'il sollicite à plusieurs reprises de Gide. Il faut enfin, si l'on suit les critères reconnus par Pierre Bourdieu et Jacques Dubois pour conquérir sa place dans les plus hautes sphères de l'institution littéraire, afficher des positions théoriques sur une conception originale de la littérature, si possible en rupture avec les modèles dominants. C'est ce que fait aussi Simenon. De ses reportages autour du monde, et particulièrement en Afrique, il rapporte sa théorie de l'homme nu, qu'il développe dans de nombreux entretiens, pour démontrer qu'il possède un véritable projet d'écrivain. Le reporter se veut anthropologue, l'écrivain veut saisir l'être humain dans son essence profonde, comme Kafka auquel il se compare lorsqu'il veut rendre « l'homme le plus vrai, c'est-à-dire le plus nu » :

[...] Les romans de l'homme habillé sont des romans de mœurs, des romans d'époque, etc. C'est l'homme dans la société, ressemblant à ce qu'il voudrait être. Ce n'est que récemment que l'on s'est occupé de l'homme tout nu, c'est-à-dire presque en dehors de la vie sociale. Kafka s'occupe de l'homme tout nu. Cela peut se passer n'importe où, n'importe quand. (dans L'Express, 6 février 1958).

11 Il a désormais choisi de passer à ce qu'il considère comme des productions semilittéraires, avant de franchir le troisième niveau, celui des « romans tout courts », qui lui assureront la considération de l'institution. Il croit naïvement qu'il pourra effacer la tache originelle de ses productions populaires, que la mobilité institutionnelle est possible, et conciliable avec ses options de marketing commercial et de séduction du public le plus large. Mais il ignore combien les discriminations restent fortes dans cette institution littéraire à l'époque encore bien séparée en deux (ou plusieurs) champs distincts, et qu'il est impossible de vouloir à la fois acquérir le capital économique et le capital culturel. Si l'on veut accéder au champ de production restreinte, la reconnaissance ne s'y acquiert pas sur des principes hétéronomes (comme l'importance des tirages) mais autonomes (la reconnaissance par les pairs, la critique). Et cette reconnaissance, Simenon peine à l'obtenir, même si des critiques attirent l'attention sur ses premiers romans policiers. Face à cette résistance, Simenon va progressivement prendre ses distances avec l'institution, en pleine lucidité.

12 Finalement, l'auteur au succès mondial qui rêva longtemps d'un prix Nobel pour lequel il fut à plusieurs reprises évoqué ne réussit jamais à combler de son vivant le manque originel. Mais Jacques Dubois parviendra cependant à le faire publier dans la Pléiade, en deux volumes de romans choisis, en 2003, bientôt suivis d'un troisième volume en 2009, 
tant les ventes furent importantes. On peut se demander si cette ultime consécration que représente l'entrée de Simenon dans la Pléiade ne correspond pas à une évolution des instances de légitimation, par une plus grande ouverture des structures traditionnelles comme l'école ou la critique universitaire. Mais il faut alors s'interroger sur les raisons qui justifient cette entrée. Sont-ce les mêmes qui ont permis à un autre auteur phare du $\mathrm{xx}^{\mathrm{e}}$ siècle, Albert Cohen, d'y accéder, ou plutôt celles qui ont permis l'entrée d'un romancier du XIXe siècle, Alexandre Dumas. Les similitudes sont sans doute plus grandes avec ce dernier : auteur méprisé par les institutions littéraires et scolaires de son temps, auteur pourtant d'une œuvre doublement importante, en quantité et par son succès public. Simenon et Dumas représenteraient cet improbable équilibre entre la production de masse et la qualité narrative et esthétique. Ce sont, pour reprendre les termes de Jacques Dubois, des auteurs qui appartiennent à une zone médiane, qui ne relève ni de l'avant-garde, ni de la littérature populaire :

Il est sûr que Simenon a fait accéder le policier, en lui intégrant des éléments psychologiques, en le dotant d'un vraisemblable sérieux, à cette littérature bourgeoise et moyenne qui a pris de plus en plus d'extension avec le siècle. [...] L'écrivain liégeois risquerait donc facilement de se diluer dans la grisaille de la zone médiane qu'il a laborieusement rejointe. Ce qui le sauve est que sa position moyenne et mitoyenne [...] atteint avec lui à une manière de comble. Toute l'entreprise simenonienne est comme tendue, de façon entêtée, vers cet accomplissement. (Dubois $1991: 12$ )

13 Pour rester dans la référence bourdieusienne, ne pourrait-on dire qu'Alexandre Dumas, dont l'œuvre se développa avec les débuts de la photographie, que Georges Simenon, qui fut un photographe passionné et passionnant, appartiennent à cet "art moyen " (Bourdieu 1965) qui se développe dès la fin du XIX ${ }^{e}$ siècle, et qui ne touche pas seulement l'art photographique, mais aussi la littérature, quand elle touche des centaines de millions de lecteurs de par le monde.

\section{Le roman policier, un roman comme les autres?}

14 Le conflit qui oppose les défenseurs du roman policier et ceux de la littérature "pure » est assez semblable à celui qui oppose les écoles littéraires, aux $\mathrm{XIX}^{\mathrm{e}}$ et $\mathrm{xx}^{\mathrm{e}}$ siècles, dans cette querelle récurrente des « Anciens » et des « Modernes ». En fait, la question ne peut pas être résolue car elle est mal posée. Vouloir comparer deux conceptions différentes n'a guère de sens, à moins qu'il ne soit possible de définir une fois pour toutes ce qu'est la littérature, et de mesurer toutes les œuvres à l'aune de cette définition définitive. Personne n'oserait s'y risquer explicitement, mais nombreux sont ceux qui s'avancent dans cette voie sans l'avouer. Jacques Dubois va cependant montrer les limites d'une telle dichotomie, à la lumière des recherches sur les paralittératures :

La littérarité, essence particulièrement élective, se trouve étendue à quantité d'écrits dont on postulait que, dans leur masse même, dans leur usage trivial également, ils étaient sans valeur et sans intérêt. Or, leur entrée dans le champ critique, même si elle se fait par une porte latérale, a pour conséquence immédiate de mettre en question l'image reçue de la littérature, la conception ordinaire de sa définition et de ses limites. Si les genres paralittéraires s'élaborent et fonctionnent suivant les mêmes structures que les productions consacrées, qu'est-ce qui justifie encore la séparation entre les deux sphères et les critères usuels de qualité ou de valeur? (Dubois 1988a : 15) 

d'inspiration, de génie pur suggérées par nombre d'écrivains et complaisamment véhiculées par les histoires littéraires. Mise en avant de la conception préalable, de la logique, de la composition fabriquée, refus du hasard et de l'intuition: il y a à la fois l'annonce, dans ce véritable art poétique, d'importantes tendances littéraires du $\mathrm{xx}^{\mathrm{e}}$ siècle : Valéry ou le Nouveau Roman mais aussi des techniques de composition du récit d'énigme. Poe, comme le dit Narcejac, «a été, en littérature, l'auteur d'une révolution identique à celle que Descartes avait réussie, en philosophie» (1975: 18). Mais c'est précisément cette systématisation de la création que Narcejac condamne, sinon dans le chef-d'œuvre de Poe, du moins dans les œuvres de ses épigones, ramenant ce type de récit, non plus à une œuvre artistique mais à une « machine à lire». synchronique, comme l'envisage erronément Narcejac, mais dans une dimension diachronique. La conception de l'esthétique évolue au fil du temps, et au prix de combats incessants des forces novatrices des « Modernes » contre les positions conservatrices des "Anciens ». La paralittérature, dans cette vision de l'objet littéraire, ne serait qu'une sorte d'antichambre où patienteraient les candidats avant de recevoir l'imprimatur de l'institution littéraire. S'il a fallu au roman plusieurs siècles pour être reconnu comme un genre noble, le roman policier, pour sa part, semblera devoir faire preuve de moins de patience.

17 Ce combat historique (dans le sens où il s'inscrit dans la durée de l'histoire) se fonde dans notre cas sur un reproche majeur avancé par tous les adversaires de cette "pseudolittérature »: «il ne s'agirait pas, rappelle Eisenzweig, d'une catégorie comportant un grand nombre de mauvais échantillons, mais bien d'une mauvaise littérature parce que constituée en catégorie » (Eisenzweig 1983a : 20). Ce qui dérange dans ces récits, ce n'est pas tellement tel ou tel roman pris individuellement, c'est l'idée qu'une catégorie entière de récits puisse se constituer selon les mêmes normes, avec une telle cohérence d'ensemble que certains auteurs pourront définir très strictement les règles à respecter pour composer ces intrigues, que d'autres écriront des romans collectifs, les contraintes narratives étant suffisamment fortes pour assurer l'unité d'ensemble. C'est la dénonciation nette, même s'il n'y eut jamais de manifeste en ce sens, de la notion de génie créateur, le démiurge exprimant par essence son univers personnel et individuel. «Perçu comme genre, dit Eisenzweig, le roman policier ne pouvait être considéré, dès l'abord, que comme une littérature dégradée » (Ibid. : 21). rigide entre différentes catégories apparait, et plus nettement dans le chef des critiques que des écrivains. En Angleterre, Chesterton écrit aussi bien des romans "classiques » que des récits policiers; en Russie, Dostoïevski emprunte des trames policières pour nombre de ses romans ; aux États-Unis, Hemingway reconnaît sa dette envers Hammett et Chandler. Mais en France, l'image que se donne l'institution littéraire l'entraîne à garder ses distances vis-à-vis d'un genre de grande diffusion, la littérature y restant encore considérée comme une activité "noble » qui ne peut que se dégrader par une diffusion importante auprès de la masse.

19 Si la question de la reconnaissance littéraire du genre policier en tant que tel par l'institution est donc affaire de temps, comme le montrent l'évolution récente du roman policier et l'attitude des écrivains à son égard, il ne s'agit cependant pas de vouloir 
justifier la qualité de chacun des ouvrages du genre. Ce qui est demandé, c'est de ne plus considérer le genre dans son ensemble et de le condamner en soi, en excipant de tel maillon plus faible de la chaîne. Il est vrai que cette littérature peut engendrer des procédés répétitifs et stéréotypés, mais en même temps, la subversion du genre est quasi inscrite dans son code générique qui impose d'aller toujours plus loin dans les innovations, jusqu'au moment où la frontière, toujours floue, entre ce qui relève du genre et ce qui y échappe est franchie. Il s'agit donc de transgresser tout en restant dans certaines limites, mais en sachant que le contrat impose la subversion. C'est ce que feront tous les grands auteurs, dès les origines, et ce rapport ambigu de fidélité/transgression est peut-être le critère qui permet d'identifier les grands textes policiers. Ceux qui subvertissent le genre de l'intérieur innovent; ceux qui restent prisonniers des règles restent des épigones besogneux. Jacques Dubois dresse ce constat à propos de Gaston Leroux :

Les grands textes de la littérature policière enfreignent les lois du genre. C'est l'effet de leur liberté créatrice autant que d'une propension à surenchérir dans le surprenant, dans l'inédit. [...] L'hypothèse est donc que certains textes policiers parmi les plus construits se distinguent par le traitement désinvolte qu'ils font subir à la structure de base et par la crise qu'ils ouvrent dans le genre dont ils se réclament. (Dubois $1988 \mathrm{~b}: 17$ )

Mais si certains auteurs émergent dans ce sous-champ, le roman policier dans son ensemble n'a jamais nié être un " objet de consommation ", par sa filiation avec les récits de colportage et les romans-feuilletons. Il s'est toujours inscrit dans un rapport direct avec son public potentiel, visant à atteindre le maximum d'audience, les tirages les plus importants. Le roman policier est dans cette logique identifié comme une catégorie à part, voire un genre, parce que c'est aussi un produit bien identifiable, bien diffusé et bien lu. C'est un produit facilement identifiable par l'acheteur potentiel. Il est en général publié dans des collections spécialisées, dont les plus célèbres, « Le Masque » et la « Série noire", sont connues de tous les publics. Couverture toujours identique, classement particulier dans les librairies et autres points de vente, tirages massifs assurent au lecteur un repérage facile. Dans ces collections, certains « indices » sont encore mis en avant: nom de l'auteur, s'il est connu du grand public (Agatha Christie, au Masque, a sa propre collection dans le catalogue), ou du héros (les noms de Maigret ou d'Arsène Lupin sont imprimés en plus grands caractères sur les couvertures que ceux de Simenon ou de Leblanc).

21 Le phénomène des séries va encore renforcer ces habitudes de lecture. Lorsqu'un personnage rencontre le succès, son auteur sera amené à multiplier ses aventures, parfois sous la pression du public. On connaît le tollé soulevé par la mort de Sherlock Holmes, au point que Conan Doyle fut obligé de le ressusciter, ou la difficulté que ressentait Maurice Leblanc à se séparer d'Arsène Lupin dont la gloire envahissante le gênait. Couvertures, séries, titres isolent donc ce type de production des autres publications romanesques, mais en permettant aussi une identification plus rapide. La critique journalistique avalise d'ailleurs cette pratique en consacrant, tant dans la presse quotidienne que dans la presse spécialisée (Le Magazine littéraire), des rubriques particulières au genre policier.

\section{Roman policier et Nouveau Roman}

Mais si le genre policier semble longtemps exclu de la littérature légitimée, certains mouvements ont montré que cette division était en train de disparaître, dès les années 
1960. Au départ, il y a cette affirmation de Ludovic Janvier : "En somme, le Nouveau Roman, c'est le roman policier pris au sérieux » (1964: 49). Très vite, les relations qui vont s'établir - que ce soit le fait de la critique ou des auteurs eux-mêmes - entre le roman policier et le courant baptisé Nouveau Roman seront exemplaires de l'évolution du roman contemporain et du regard porté autant sur le roman reconnu comme littéraire que sur le roman policier. La boutade de Ludovic Janvier est révélatrice de cet état de fait : elle affirme la reconnaissance du Nouveau Roman par rapport au roman policier tout en situant implicitement ce dernier hors du champ littéraire. L'ambiguïté du critique n'est ici que le reflet des sentiments ambivalents des romanciers face à un genre dans lequel ils se reconnaissent tout en s'en distanciant, par le recours à la parodie entre autres.

Toutes les critiques, souvent virulentes, adressées au Nouveau Roman sont similaires à celles adressées au roman policier. Personnages stéréotypés, sans psychologie, situations invraisemblables, structure rigide brimant la «liberté d'action» des héros, manque d'humanité, les mêmes termes sont employés pour dénoncer le roman policier et le Nouveau Roman, tant il est vrai que ces deux types de récits remettent en cause le même dogme de la littérature classique basé sur les valeurs d'expression et de représentation, et sur la notion de vraisemblable. Mais si Hanna Charney reconnaît ces similitudes, elle souligne cependant une différence essentielle :

[Dans le Nouveau Roman,] l'enquête, en effet, n'aboutit presque jamais [...]: les indices n'indiquent rien, les objets mêmes sont trompeurs [...]. Les souvenirs ne se recoupent pas [...], le but de l'enquête même est grotesque [...]. Dans le roman policier, au contraire, on est assuré d'atteindre la solution. (Charney 1972 :21)

Ces parallélismes entre Nouveau Roman et roman policier vont apparaître à la fois de manière ponctuelle, à travers les figures de l'errance, du labyrinthe, de l'étranger, de l'enquêteur, de l'énigme, mais aussi dans un rapprochement plus systématique et nullement dû au hasard, au point que Ludovic Janvier consacrera un chapitre de son étude au « Point de vue du policier» (1964: 39-49). Sans prétendre que les intentions de ces deux types de récits sont les mêmes, les convergences multiples permettent cependant de dire que certains de leurs objectifs sont communs. Passage de Milan de Michel Butor comporte une scène importante de meurtre et Degrés relève de l'enquête policière, mais c'est dans L'Emploi du temps que Butor est le plus proche du roman policier. Todorov, dans sa « Typologie du roman policier » (1971), a montré comment ce roman est en même temps une réflexion sur le genre policier. Un enquêteur sur les traces de son passé, à la recherche de la vérité, il s'en trouve aussi un, tout autant CEdipe que Thésée, dans cet autre roman à références mythiques de Robbe-Grillet, Les Gommes. Ce premier roman publié de Robbe-Grillet (1962) utilise explicitement la trame policière. Ainsi qu'il est dit, avec une ingénuité feinte ou réelle, au dos de la couverture : "Cette œuvre, aussi captivante que le meilleur roman policier, est une date dans la littérature contemporaine. » Bruce Morrissette considère d'ailleurs cette hypothèse de lecture avec un certain dédain : "Une lecture très superficielle pourrait à la rigueur faire passer Les Gommes pour un ingénieux roman policier à dénouement-surprise » (Morrissette 1962: 281). Le roman policier est donc plutôt considéré comme une structure que le Nouveau Roman va investir pour produire un nouveau type de texte, ainsi que l'avance Stefano Tani :

Ainsi les règles codifiées par Poe, exploitées depuis par le roman policier britannique, une fois libérées de la tyrannie de la centralisation des genres, et 
rendues au libre circuit de l'écriture, se sont révélées capables d'engendrer de nouveaux produits hybrides fort originaux. (Tani 1982 : 49) dans ce même article ne pas croire aux catégories et entretenir avec les genres littéraires des rapports pervers, il cloisonne pourtant aussitôt les romans policiers dans une catégorie précise dont il exclut, par après, tous les auteurs qu'il apprécie. Ayant très justement montré l'envahissement progressif de la psychologie dans les récits de Simenon, il loue Le Meurtre de Roger Ackroyd et les récits de Hammett et de Chandler, mais en les considérant comme "des marginaux du roman policier». Curieuse pratique, marquée de présupposés idéologiques, consistant à enfermer dans un genre les récits qu'on n'aime pas et à en extraire, pour les sauver, ceux qu'on apprécie. Robbe-Grillet rejoint en quelque sorte les plus ardents défenseurs de la littérature réaliste de la fin du $\mathrm{XIX}^{\mathrm{e}}$ siècle pour lesquels Eisenzweig avait déjà observé le même mécanisme d'exclusion. C'est le piège qu'évitera Umberto Eco dans Le Nom de la rose dont le prière d'insérer indique que «c'est d'abord un grand roman policier pour amateurs de criminels hors pair ».

\section{Un genre bien identifié, ouvert aux hybridations}

L'apport du Nouveau Roman a eu son importance en ce qu'il a modifié le regard porté sur le roman policier. Même si les remarques de Robbe-Grillet témoignent encore d'une réticence, en partie inavouée, les critiques se basent désormais sur des critères d'ordre formel ou esthétique et ont abandonné le jugement moral pour se cantonner plus strictement dans le champ littéraire. Il semble que l'appropriation de certaines techniques du récit d'énigme par des œuvres jugées comme relevant de la littérature, que le goût déclaré pour ce genre par certains auteurs reconnus aient enfin permis au roman policier de sortir d'une forme de ghetto.

Ceci est d'ailleurs facilité par le fait que certains chercheurs vont jusqu'à considérer le roman policier comme l'archétype même du roman. L'énigme, cette forme primitive, se révèle de manière exemplaire dans les récits policiers, faisant de ceux-ci l'image archétypale d'une littérature désormais consciente de sa signification herméneutique. Le texte policier ne serait ainsi qu'un palimpseste à déchiffrer, sa lecture étant un acte essentiellement herméneutique, comme l'ont montré, de manière théorique, Jacques Dubois, et sous forme romanesque, Umberto Eco dont on sait que le livre, dans Le Nom de la rose, sous son apparence réelle et de manière métaphorique, est à la fois le centre et la solution d'une intrigue policière située dans une bibliothèque de moines copistes. C'est ce qui permet à Dubois d'avancer l'idée que « le récit d'énigme semble le comble de tout acte narratif » (1984: 116), ce que Peter Brooks avait déjà affirmé : 
Le roman policier peut de la sorte être vu comme modèle du récit en général, et remarquable en ce qu'il démontre et dramatise ouvertement le rapport entre histoire et récit. Le roman policier est peut-être en fait, de tous les genres, le plus littéraire, en ce qu'il dévoile la structure de tout récit, et particulièrement sa prétention de retrouver la trace d'événements ayant déjà eu lieu. (Brooks 1992 : 477)

29 Le policier serait ainsi un genre où CEdipe est roi, pour reprendre l'expression de Dubois (1992), parce qu'il perpétue une tradition herméneutique inscrite au cœur de tout acte narratif, mais également parce qu'il constitue le modèle de référence de tout un courant de la modernité littéraire. Si le genre policier existe, ce n'est donc pas dans un carcan monolithique et figé, mais sous des formes très variées, et avec une possibilité de transformation assez éloignée de l'image répétitive et réductrice appliquée généralement aux diverses expressions du champ paralittéraire. Il peut ainsi se combiner dans différentes formes d'hybridation avec d'autres genres et catégories romanesques.

Ce fut le cas avec l'école du Nouveau Roman, ce l'est encore aujourd'hui. Le succès du roman d'Eco, Le Nom de la rose, ouvertement déclaré comme une enquête policière par son auteur, en est la preuve. Patrick Modiano, dans ses intrigues, recourt fréquemment à des histoires policières, à des enquêtes; René Belletto qui est publié dans la collection « Textes » de Flammarion ou chez P.O.L., maisons d'édition « littéraires » s'il en est, reçoit en 1984 le grand prix de littérature policière pour son roman Sur la terre comme au ciel et le prix Femina en 1986 pour L'Enfer. Alain Demouzon, Jean Vautrin (bien vite récompensé par un prix Goncourt), Daniel Pennac ou Pierre Magnan font, eux, le trajet inverse, passant du roman policier à la littérature générale, en restant parfois chez le même éditeur ou en recourant à divers éditeurs selon le genre abordé. Daniel Pennac publie ainsi les deux premiers volumes de sa série des Malaussène à la "Série noire » avant de les continuer dans la « Blanche », puis d'abandonner le genre policier.

31 Ces franchissements des frontières sont aujourd'hui reconnus par les discours critiques et par les auteurs eux-mêmes, comme en témoignent les nombreux débats autour du roman de Joël Dicker, La Vérité sur l'affaire Harry Quebert, qui a reçu le Grand Prix du roman de l'Académie française en 2012. Et cela quand la "Série noire " se démarque des attributs anciens du genre, quand les auteurs ne se revendiquent plus du roman policier mais s'inscrivent dans le cadre plus large de la "littérature noire", pour manifester leur volonté de sortir de catégories génériques trop formatées, quand les chercheurs euxmêmes préfèrent analyser le roman noir plutôt que le roman policier (Levet 2006).

Cela pose la question de l'existence, aujourd'hui, du genre comme entité autonome, même si des collections comme "Rivages Noir» ou "Actes Noir» attestent du succès grandissant de certains textes. Mais c'est peut-être l'arbre des best-sellers qui cache la forêt d'un genre en voie d'évaporation ou d'assimilation à une catégorie plus large.

Ainsi, la distance, des points de vue stylistique, thématique, narratif, apparait moins grande qu'avant entre le roman légitimé et la paralittérature policière, des auteurs reprenant les techniques de l'une ou l'autre catégorie ou passant d'un genre à l'autre au fil des livres qu'ils publient, comme le reconnait, par exemple, un Jean Echenoz. Au point que les études récentes ne ressentent presque plus le besoin de justifier l'intégration du genre policier au continent romanesque. L'essai récent d'Isabelle-Rachel Casta (2012) soulève la question des relations entre policier et philosophie, policier et mythes anciens, entre polar et cinéma, polar et chanson, mais sans consacrer d'entrée spécifique à la question de la dimension littéraire du policier. Comme si la question n'était plus de mise. 
Dans un autre registre, le roman policier le plus classique, comme les enquêtes d'Agatha Christie, sera l'occasion, ou le prétexte, à réflexion savante sur l'art de l'interprétation ou la critique de la lecture, ainsi qu'en atteste l'essai de Pierre Bayard, Qui a tué Roger Ackroyd? (1998), publié chez un éditeur des plus légitimés, Minuit. La sociologie ellemême peut se revendiquer d'une filiation avec le roman policier, non plus pour le prendre comme objet d'étude en soi, mais pour rechercher les parentés épistémologiques entre les figures des enquêteurs dans les romans policiers et d'espionnage et les démarches du sociologue. C'est à cet exercice que s'est essayé Luc Boltanski en 2012.

Mais s'il y a une tendance à moins dissocier roman "classique » et policier, cette catégorie garde cependant son autonomie: les collections spécifiques comme "Le Masque » ou la "Série noire » existent toujours, et reviennent parfois au modèle éditorial des origines. Et des dossiers spécifiques lui sont encore spécialement dédiés: par exemple, le numéro d'avril 2014 du magazine Lire s'intitule "Spécial Polar: les 10 meilleurs ", au moment même où Le Monde sort un supplément hors série titré « Polar. Le triomphe du mauvais genre ».

Malgré ces contre-exemples, permettant de rappeler que cette catégorie spécifique du champ littéraire n'a pas encore disparu dans les représentations mentales et les classifications des éditeurs, le genre pourrait donc sembler se diluer peu à peu dans la sphère plus englobante du roman, devenu aujourd'hui le genre dominant du champ littéraire. Pour certains, l'insertion du roman policier dans la paralittérature arriverait à son terme, celle-ci ne servant que de purgatoire avant une reconnaissance institutionnelle. Il y aurait donc des phases transitoires d'intronisation, avant qu'un genre nouveau ne soit intégré dans le système littéraire, de la même manière que le roman lui-même a mis plusieurs siècles avant d'être reconnu comme un genre noble. Mais ce point de vue irrite certains thuriféraires des genres paralittéraires qui préfèrent rester enfermés dans leur citadelle, plutôt que de voir le récit policier perdre ses qualités propres et se diluer dans le vaste océan de la littérature générale. Les deux mouvements s'observent donc conjointement: défense d'un territoire bien balisé dans les limites du genre policier tel que défini dans ses diverses sous-catégories, ouverture à des formes d'hybridation multiples et intégration au sein d'un genre romanesque indifférencié.

Ce mouvement d'intégration ne ferait aussi que rejoindre une tendance à la disparition des frontières, lesquelles ont toujours été nettement moins marquées dans la tradition anglo-saxonne où la littérature mainstream était moins dévalorisée, et les auteurs moins vite catalogués dans un genre donné. Mais dans d'autres littératures européennes, cette séparation est aussi moins nette: un Manuel Vasquez Montablan en Espagne, un Carlo Emilio Gadda ou un Antonio Tabucchi en Italie, un Jose Cardoso Pires au Portugal sont ainsi passés par le genre policier ou ont reconnu sans aucune réserve choisir cette structure romanesque ou cette thématique noire comme trame narrative la plus appropriée pour mettre en récit le sujet d'un de leurs romans. L'hypothèse d'une hiérarchisation des genres qui serait propre à la littérature française est peut-être à interroger, même si elle semble s'estomper. C'est ainsi que si l'on remonte aux origines de la littérature fantastique, en Angleterre, en Allemagne, en Russie ou en Amérique, on sait qu'elle se développa au départ des auteurs les plus légitimes, dans une filiation avec la littérature gothique, et sans connaître cette forme de marginalisation qu'elle connaîtra en France. Il semble en avoir été de même, et de manière encore plus exacerbée, avec le roman policier français. 


\section{BIBLIOGRAPHIE}

Assouline, Pierre, 1992, Simenon. Biographie, Paris, Julliard.

Bayard, Pierre, 1998, Qui a tué Roger Ackroyd ?, Paris, Minuit.

Boltanski, Luc, 2012, Énigmes et complots. Une enquête à propos d'enquêtes, Paris, Gallimard, coll. « NRF Essais ».

Bourdieu, Pierre, 1965, Un art moyen. Essai sur les usages sociaux de la photographie, Paris, Minuit, coll. « Le sens commun ».

-, 1991, « Le champ littéraire », Actes de la recherche en sciences sociales, n 89, p. 3-46.

Brecht, Bertolt, 1970, Les Arts et la Révolution, précédé de Notes sur le travail littéraire. Articles sur la littérature, Paris, L'Arche.

Brooks, Peter, 1980, « Un rapport illisible : Coeur des ténèbres », Poétique, nº 44, p. 472-489.

Casta, Isabelle-Rachel, 2012, Pleins feux sur le polar, Paris, Klincksieck, coll. « 50 questions ».

Charney, Hanna, 1972, « Pourquoi le "Nouveau Roman" policier? ", The French Review, vol. 46, n 1, p. 17-23.

Couégnas, Daniel, 1992, Introduction à la paralittérature, Paris, Seuil, coll. « Poétique ».

Dubois, Jacques, 1984, «Indicialité du récit policier », dans C. Gothot-Mersch (dir.), Narration et interprétation, Bruxelles, Publications des Facultés universitaires Saint-Louis, p. 115-128.

-, 1988a, L'Institution de la littérature. Introduction à une sociologie, Bruxelles, Labor, coll. « Média ».

-, 1988b, « Rouletabille et l'aventure mentale », Les Cahiers des paralittératures, n² 2, p. 17-29.

-, 1991, «Situation de Simenon », Traces, $\mathrm{n}^{\circ}$ 3, p. 9-18.

-, 1992, Le Roman policier ou la Modernité, Paris, Nathan, coll. « Le texte à l'œuvre ».

Eisenzweig, Uri, 1983a, Autopsies du roman policier, Paris, Union générale d'édition, coll. « 10/18 ».

-, 1983b, « Alain Robbe-Grillet. Entretien », Littérature, nº 49, p. 16-22.

Genette, Gérard, 1991, Fiction et diction, Paris, Seuil, coll. « Poétique ».

Janvier, Ludovic, 1964, Une parole exigeante. Le Nouveau Roman, Paris, Minuit.

Levet, Natacha, 2006, Le Genre, entre pratique textuelle et pratique sociale : le cas du roman noir français (1990-2000), Thèse de doctorat en littérature française, Limoges, Université de Limoges, [En ligne], http://epublications.unilim.fr/theses/2006/levet-natacha/html/index-frames.html.

Lomnitz, Sacha, 1999, « Le Poulpe, Le Pen et le roman populaire », Critique, vol. 55, nº 624, p. $445-456$.

Morrissette, Bruce, " Clefs pour Les Gommes », postface au roman d'Alain Robbe-Grillet, Les Gommes, Paris, Union générale d'édition, coll. « 10-18», p. 281.

Narcejac, Thomas, 1975, Une machine à lire : le roman policier, Paris, Denoël, coll. « Médiations ». Poe, Edgar, [1846] 1871, «Philosophy of Composition », Graham's Magazine, trad. fr. de Charles Baudelaire, « La genèse d'un poème », Paris, Michel Lévy frères, p. 334-371. 
Robbe-Grillet, Alain, [1953] 1962, Les Gommes, Paris, Union générale d'édition.

Simenon, Georges, 1981, Mémoires intimes suivis du livre de Marie-Jo, Paris, Presses de la Cité.

Tani, Stefano, 1982, « Le détective écartelé », Diogène, $\mathrm{n}^{\circ}$ 120, p. 26-51.

Todorov, Tzvetan, 1971, « Typologie du roman policier », dans Poétique de la prose, Paris, Seuil, p. 55-65.

\section{NOTES}

1. Repris sur le site en ligne de Paris Match, «Un entretien avec Pierre Lemaître. Rencontre avec le Goncourt 2013 ", http://www.parismatch.com/Culture/Livres/Pierre-Lemaitre-rencontreavec-le-Goncourt-2013-535422, consulté le 5 mars 2015.

2. Voir Gérard Genette (1991 : 7-40).

3. Toutes les citations de ce paragraphe proviennent des p. 16-20.

\section{RÉSUMÉS}

De nombreux auteurs policiers se font aujourd'hui reconnaître par des publications plus légitimées, ce qui pose la question de l'organisation interne du genre romanesque, et de la position ambiguë du roman policier au sein de l'institution littéraire. Si l'exemple de Simenon est significatif de ces jeux de distinction, les formes de légitimation sont plus aisées depuis que le Nouveau Roman a affiché, non sans ambiguïté, son intérêt pour le genre policier. Même si les frontières sont aujourd'hui plus poreuses, la littérature française reste davantage cloisonnée que d'autres littératures européennes ou américaines.

Many crime fiction writers nowadays attempt to get noted through more "literary" works, which raises the question of the internal organization of the novelistic genre, and the ambiguous position of the detective novel within the literary institution. Simenon's example is typical of these types of distinctions, even if claims of legitimacy have become more acceptable since the Nouveau Roman's display of interest, albeit quite ambiguous, for crime narratives. Although the boundaries between genres are seen today as less rigid, French literature remains more compartmentalized than American or other European literatures.

\section{INDEX}

Mots-clés : roman policier, littérature populaire, paralittérature, légitimité, genre littéraire Keywords : detective novel, popular literature, legitimacy, literary genre 


\section{AUTEUR}

\section{MARC LITS}

Université catholique de Louvain, Observatoire de recherche sur les médias et le journalisme 